\title{
Eye color prediction using the IrisPlex system: a limited pilot study in the Iraqi population
}

\author{
Nihad A.M Al-Rashedi ${ }^{*}$, Amar Mousa Mandal and Laith AH ALObaidi
}

\begin{abstract}
Background: Forensic DNA phenotyping has gained momentum in the recent past due to the prediction of externally visible characters (EVCs) from the biological sample. The most common phenotypes like eye, hair, and skin color are predicted from the biological samples using a web-based system called IrisPlex. Based on six genetic SNPs, the IrisPlex system is developed and validated for its prediction accuracy in diverse ethnic groups worldwide. In previous studies, this system proved to have significant prediction accuracy. The EVCs vary substantially based on different geographical locations. Hence, the objective of this study was to validate the accuracy of the IrisPlex system in predicting the eye colors in the Iraqi population.

Methods: Six genetic single-nucleotide polymorphisms SNPs (HERC2-rs12913832, OCA2- rs1800407, SLC24A4rs12896399, SLC45A2- rs16891982, TYR-rs1393350, and IRF4- rs12203592) in 58 Iraqi subjects were performed using Sequenom MassARRAY Genotyping. According to Liu et al., a predicted probability of 0.7 was considered as the threshold.

Results: Participants in this study of brown color were observed in $44.83 \%$, intermediate in $43.1 \%$, and blue in $12.07 \%$. Completely predictive accuracy is obtained in 1 ; we observed the AUC at threshold 0.7 was 0.91 for brown, 0.79 for blue, and 0.60 for intermediate eye color. The sensitivity was $42.85 \%$ for blue, $0 \%$ for intermediate eye color, and $100 \%$ for brown-colored eye. Specificity was 100\% for blue, 100\% for intermediate, and 78.13\% for brown eye color.

Conclusion: Hence, it was concluded that the prediction accuracy of the IrisPlex system for blue and brown color eye in the Iraqi population is significant in the studied population size. However, a pivotal study with larger sample size is required to represent the prediction accuracy of the IrisPlex system in the whole Iraqi population.
\end{abstract}

Keywords: Eye color, Genetic markers, MassARRAY SNP genotyping, IrisPlex, Iraq

\section{Background}

Externally visible characteristics (EVCs) prediction using DNA has opened a new avenue in forensic genetics. The EVCs like eye, hair, and skin color prediction from the biological crime scene materials can reduce the number of suspects where the perpetrators are unknown to the investigators of the case. Variation in the eye color is dependent on the number and distribution of stromal

\footnotetext{
* Correspondence: nhidaee@mu.edu.iq; nihadalrashedi1@gmail.com; nhidaee@mu.edu.iq

Department of Biology, College of Science, Al Muthanna University, Samawah, Iraq
}

\section{Springer Open}

melanocytes (Sulem et al. 2007). This phenotypic variation in humans is due to the difference in the corresponding genotype subject to the geographical distribution of populations. Several genes like $S L C 45 A 2$, TYR, TYRP1, ASIP, HERC2, OCA2, MC1R, SLC24A5, KITLG, IRF4, SLC24A4, and TPCN2 are associated with eye, hair, and skin color in the European population (Branicki et al. 2011; Frudakis et al. 2003; Han et al. 2008; Liu et al. 2009; Mengel-From et al. 2010; Pneuman et al. 2012). Out of these genes, ASIP, IRF4, HERC2, OCA2, TYR, TYRP1, SLC24A4, SLC45A2, and SNPs in these genes are associated with eye color and thereby 
used in the prediction of eye color studies (Pośpiech et al. 2012; Ruiz et al. 2013; Spichenok et al. 2011; Kayser and Schneider 2009; Sulem et al. 2008; Walsh et al. 2011a; Walsh et al. 2011b; Walsh et al. 2012). The color of the eye is majorly classified into three types, namely blue, brown, and intermediate (Liu et al. 2009; Walsh et al. 2012). Hence, the variability in eye color constitutes a distinguishing trait, thereby used majorly in the forensic investigation (Pośpiech et al. 2012; Walsh et al. 2012).

Polymorphism in the evolutionary conserved HERC2 and OCA2 gene is thought to contribute substantially in the human iris color differentiation. It was reported that the extent of expression of the OCA2 gene (pigmentation gene) is regulated by polymorphism rs12913832 on HERC2 locus (Visser et al. 2012; Eiberg et al. 2008). The rest of all the genes (SLC45A2, TYR, SLC24A4, and IRF4) seem to contribute less in the prediction of eye color and differ with populations (Ruiz et al. 2013; Walsh et al. 2012; Allwood and Harbison 2013; Branicki et al. 2009). Several models have been proposed in the recent past for the prediction of eye color (Liu et al. 2009; Ruiz et al. 2013; Spichenok et al. 2011; Allwood and Harbison 2013; Hart et al. 2013).

The IrisPlex (Liu et al. 2009) is a genetic web tool developed based on six Single-nucleotide polymorphisms (SNPs) and has a prediction accuracy of 93 and $91 \%$ for brown and blue color eye, respectively, in Europeans. However, for an intermediate color eye, the prediction accuracy was reported to be $73 \%$ with a sensitivity of $1.1 \%$. Six SNPs from six genes, rs12913832 (HERC2), rs1800407 (OCA2), rs12896399 (SLC24A4), rs16891982 (SLC45A2), rs1393350 (TYR), and rs12203592 (IRF4), reported to contribute significantly to eye color (Liu et al. 2009). This IrisPlex system was validated by different laboratories independently for sensitivity and specificity (Walsh et al. 2011a).

The prediction accuracy of these web-based tools is highly population-specific, as diverse studies reported variation in allele frequencies between populations (Dario et al. 2015; Yun et al. 2014; Franssen and Coppens 2008). For the prediction of eye color, Liu et al. (Liu et al. 2009) have developed the following formula which is widely used by the researchers and the IrisPlex system is developed based on it.

$$
\begin{aligned}
& \pi_{1}=\frac{\exp \left(\alpha_{1}+\sum \beta\left(\pi_{1}\right)_{k} x_{k}\right)}{1+\exp \left(\alpha_{1}+\sum \beta\left(\pi_{1}\right)_{k} x_{k}\right)+\exp \left(\alpha_{2}+\sum \beta\left(\pi_{2}\right)_{k} x_{k}\right)} \\
& \pi_{2}=\frac{\exp \left(\alpha_{2}+\sum \beta\left(\pi_{2}\right)_{k} x_{k}\right)}{1+\exp \left(\alpha_{1}+\sum \beta\left(\pi_{1}\right)_{k} x_{k}\right)+\exp \left(\alpha_{2}+\sum \beta\left(\pi_{2}\right)_{k} x_{k}\right)}, \text { and } \\
& \pi_{3}=1-\pi_{1}-\pi_{2} .
\end{aligned}
$$

These formulas are based on multinomial logistic regression, where $\pi_{1}, \pi_{2}$, and $\pi_{3}$ are the probability of getting brow, blue, and intermediate eye color, respectively.
Every individual was categorized into brown, blue, or intermediate eye color as per the predicted probability derived from the above formulae. A threshold predicted the probability of 0.7 is used for the categorization of eye colors universally. Beyond the threshold value, it is regarded as unidentified or imprecise phenotype or unidentified genetic determinant.

To our knowledge, no previous studies have been reported on the validation of the IrisPlex system in the Iraqi population. Hence, the objective of this study was to find out the prediction accuracy of the IrisPlex system by using six SNPs in an Iraqi population sample.

\section{Materials and methods}

Collection of samples, DNA extraction, and quantification In this study, 58 Iraqi subjects were included, out of which 40 were men and 18 were women. This study was approved by the ethics committee of the Al-Muthanna University (Document number 2032 on 10/5/2018). All participants provided signed informed consent. The color of the eyes was graded as per the percentage of pigmentation based on the scores and color charts reported by Franssen L et al. (2008) (Valenzuela et al. 2010). The photographs of each subject's eyes were taken for confirmation and to avoid bias (Supplemental information SU2 Fig 1). The subjects were classified into blue, intermediate, and brown according to the eye colors. Individuals with green eyes (lighter phenotype), hazel eyes (darker phenotypes), and individuals having a combination of two or more pigments in the iris were categorized as intermediate. Subjects with blue color including all shades of blue were considered as blue eye and individuals with brown color including all brown color shades were considered as the brown eye. Blood samples were collected from all the subjects and DNA was extracted from the blood by automatic Magtration System 12GC plus, using MagDEA DNA 200 (GC) Kit as per the manufacturer's instruction.

\section{SNP genotyping}

MassARRAY SNPs genotypes of six genetic variants from six genes (HERC2- rs12913832, OCA2- rs1800407, SLC24A4- rs12896399, SLC45A2- rs16891982, TYRrs1393350, and IRF4- rs12203592) were used as predictors to determine eye color. All these SNPs were genotyped as per MassARRAY genotyping protocol. Details of the markers and primer sequences are given in Supplemental information SU1 Table 1. The cleaned products were analyzed by using a MassARRAY Compact mass spectrometer with the TYPER software. The samples were auto-clustered and cluster plots were checked for any outliers (Supplemental information SU2 Fig 2). The samples were run in duplicates. 


\section{Model-based prediction of eye color and evaluation of accuracy}

The eye color prediction model of the IrisPlex system was developed by Liu et al. (Liu et al. 2009) and aimed to estimate three prediction probability values for each of the three categories of phenotype (blue, intermediate, and brown) were developed (Supplemental information table-2). The descriptive analysis and overall prediction accuracy of the model were validated by using SPSS 21.0 (SPSS Inc., Chicago, IL, USA). The area under the curve (AUC) is the integral of the receiver operating characteristics (ROC) curve. The ROC value of 0.5 is a complete absence of prediction and a value of 1.0 is an accurate prediction. Moreover, sensitivity and specificity of the model were calculated according to Liu et al. (Liu et al. 2009), the threshold of 0.7 was the probability for categories blue, intermediate, and blue eye color as suggested by Liu et al., probability value $>0.7$ was considered positive, and $<0.7$ was predicted as undefined.

\section{Result}

\section{Baseline characteristics}

In this study, 58 Iraqi nationals participated, out of which $40(69 \%)$ were males and 18 (31\%) were females (Table 1). The mean age of the participants was $26.13 \pm 8.57$ years. The frequency of observed intermediate eye color in the studied sample was $43.10 \%$ (25 samples), the frequency of observed brown eye color was $44.83 \%$ (26 samples), and the frequency of observed blue color eye was $12.07 \%$ (07 samples). The subjects were categorized into the eye colors regardless of their intensity and when the color of the eyes was homogenous (Supplemental information SU1 Fig 1).

\section{Accuracy prediction of the IrisPlex model}

This study was evaluated by the blue, intermediate, and brown color eye prediction by IrisPlex system which matched with the observed eye color at threshold 0.7 threshold in the Iraqi population. Prediction accuracy shown by the AUC (Fig. 1) was 0.79, 0.91, and 0.60 for blue, brown, and intermediate eye color, respectively (Table 2).

The sensitivity was very high for brown eye color $100 \%$ and low for blue eye color $42.85 \%$. The lack of sensitivity was obtained for intermediate eye color, which means that no one of the analyzed intermediate eyed individuals was predicted correctly.

The specificity values obtained were very high for both blue and intermediate eye color $100 \%$, which means that the prediction of negative-blue and intermediate eye match among all samples was corrected, while the prediction of negative-brown eye match was $78.13 \%$ at threshold 0.7 (Table 2).

The positive predictive value (PPV) and negative predictive value (NPV) were calculated according to Liu et al. using the prediction threshold at the $\geq 0.7$ level. At 0.7 threshold, PPV and NPV for blue eye color were 100 and $92.7 \%$, respectively. For brown color, PPV and NPV were 78.7 and $100 \%$, respectively. The PPV value was not determined for intermediate eye color due to the fact that means no cases the observed intermediate eye color was predicted as intermediate and NPV was 56.89.

At threshold 0.7, the association of gender and eye color prediction accuracy by using the six IrisPlex SNPs. In comparison, the sensitivity was $66.6 \%$ for blue eye color in males and $25 \%$ in females. The specificity for brown eye color was $84.6 \%$ for females and $73.6 \%$ for males. Prediction of the positive-brown eye color match among male samples was $80.7 \%$ and $71.4 \%$ for females.

Table 1 Baseline characteristics of participated Iraqi subjects

\begin{tabular}{|c|c|c|c|}
\hline Baseline characteristics & & $N$ & Percentage \\
\hline \multirow[t]{3}{*}{ Gender } & Male & 40 & 69 \\
\hline & Female & 18 & 31 \\
\hline & Total & 58 & 100 \\
\hline Age in years (mean $\pm S D$ ), minimum, maximum & $26.13 \pm 8.57,8,58$ & & \\
\hline Ethnicity & Iraqi & & \\
\hline Systemic ill ness & Nil & & \\
\hline Eye disorders if any & Nil & & \\
\hline \multicolumn{4}{|l|}{ Observed eye color } \\
\hline & & Frequency & Percentage \\
\hline \multirow[t]{4}{*}{ Valid } & Intermediate & 25 & 43.10 \\
\hline & Brown & 26 & 44.83 \\
\hline & Blue & 07 & 12.07 \\
\hline & Total & 58 & 100 \\
\hline
\end{tabular}




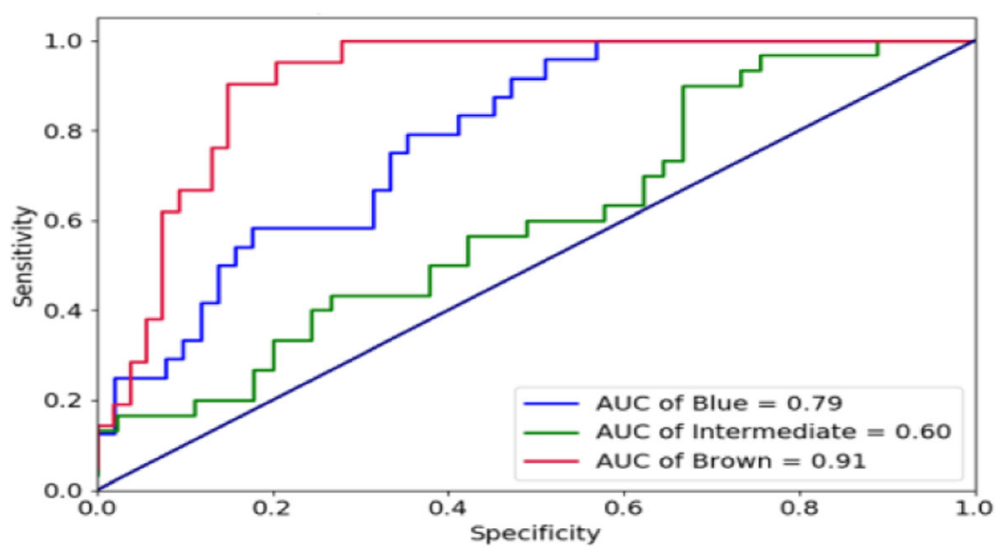

Fig. 1 Receiver operating characteristic curve analysis of 58 Iraqi samples using IrisPlex model

Prediction of the negative-blue eye match was $97.3 \%$ for males and $82.3 \%$ for females and negative intermediate eye match in males was $60 \%$ and $50 \%$ for females (Supplemental information SU1 Table-4).

\section{Discussion}

In this study, the probability of prediction of eye color by IrisPlex system matches with the observed eye color at a probability threshold value of 0.7 as per Liu et al. (Liu et al. 2009). This might be due to genotypic variance in different populations around the globe (Sulem et al. 2007; Valenzuela et al. 2010). A study on 6168 Dutch European population was carried out for the accurate prediction of the blue and brown eye by using the IrisPlex system (Walsh et al. 2011b). In this study, six genetic SNPs, namely HERC2- rs12913832, OCA2rs1800407, SLC24A4-rs12896399, SLC45A2 (MATP)rs16891982, TYR -rs1393350, and IRF4-rs12203592, were used like our study. But allele balance, for example for allele T of rs12896399 in its heterozygote state and allele C of rs16891982 in its homozygote, could not be achieved completely. However, little imbalance did not affect the genotyping accuracy in this study. The reported sensitivity of the model in this study was significantly higher compared to those previously reported autosomal SNP multiplexes included for human identification (Sanchez et al. 2006; Dixon et al. 2005). In this study, the sensitivity of the model was found to be $100 \%$ for the brown and $42.85 \%$ at threshold 0.7 for the blue color eye. As per this study, intermediate eye color was quite challenging to predict using the above SNPs and prediction model.

In our present study, $42.8 \%$ of blue eye prediction by the IrisPlex system matched with the observed eye color and for brown color eye, $100 \%$ match with the IrisPlex prediction at threshold 0.7 was achieved. In another study, the utility of the IrisPlex system was studied in a North American cohort (Dembinski and Picard 2014). In this study, with adjusted parameters, the prediction accuracy was found to be 93 and $42 \%$ for blue and brown eye color, respectively, at the prediction threshold of 0.7 . However, at 0.5 threshold value, the accuracy was found to be 95 and $58 \%$ for blue and brown color eye, respectively. The prediction accuracy for intermediate eye color was found to be $11 \%$ at threshold value 0.7 and $19 \%$ at 0.5 threshold. However, at both the thresholds, intermediate eye color did not produce any true positive prediction while using original frequencies. There was a lesser number of incorrect predictions in this study. Overall, the adjusted parameter model resulted in a lesser error rate for the blue and brown color eye at both probability thresholds (0.7 and 0.5$)$. In another study (Kastelic et al. 2013), the IrisPlex system was validated using 6 SNPs in a cohort of 105 Slovenian population.

Table 2 Eye color prediction accuracy using 6 genetic markers in the Iraqi population

\begin{tabular}{llll}
\hline Parameter & \multicolumn{1}{l}{ Color } & & Intermediate \\
\cline { 2 - 4 } & Blue & 0.60 & Brown \\
\hline Area under the receiver operating characteristic curve (AUC) & 0.79 & 0.91 & 100.0 \\
Sensitivity (\%) & 42.85 & 100.0 & 78.13 \\
Specificity (\%) & 100.0 & Nan* & 78.79 \\
Positive predictive value (\%) & 100.0 & 56.89 & 100.0 \\
Negative predictive value (\%) & 92.72 & & \\
\hline
\end{tabular}


In this study, the prediction accuracy of the system was found to be 96,91 , and $79 \%$ for blue, brown, and intermediate eye color, respectively. The sensitivity of the prediction model was found to be $93.6 \%$ (blue color), $58.1 \%$ (brown), and 0\% (intermediate color). Similarly, the specificity was reported to be $93.1,89.2$, and $100 \%$ for blue brown and intermediate eye color.

The six genetic SNPs, HERC2- rs12913832, OCA2rs1800407, SLC24A4- rs12896399, SLC45A2- rs16891982, TYR-rs1393350, and IRF4- rs12203592, reported to be associated significantly with the prediction of human eye color in diverse populations are included in IrisPlex web tool (Liu et al. 2009; Walsh et al. 2011a; Walsh et al. 2011b; Walsh et al. 2012). Out of all the above genetic SNPs, HERC2- rs12913832 reported as the most profound genetic predictor of human eye color (Liu et al. 2009; Mengel-From et al. 2010; Walsh et al. 2012; Branicki et al. 2009). This genetic SNP reported having strongly associated with blue eye color (Kastelic et al. 2013). A similar report was also published recently by Freire-Aradas et al. (Freire-Aradas et al. 2014). In their study, five SNPs exhibited high accuracy for brown and intermediate eye color compared to the report published by Liu et al. (Liu et al. 2009). The model developed by this study had a better goodness-of-fit curve and all study participants with brown eye color were predicted correctly by the IrisPlex system. HERC2- rs12913832 is also reported to play an important role in the prediction of hair and skin color (Branicki et al. 2011; Branicki et al. 2009).

In the table (Supplemental information SU2 Table S4), the difference in the eye color accuracy between males and females was notable in the blue eye color, where the sensitivity was $66.6 \%$ of the blue eye color in males and $25 \%$ in females. Our results garnered from this pilot study were consistent to demonstrate an association between sex and iris color in our population sample as it was proposed by Martinez-Cadenas et al. (MartinezCadenas et al. 2013). However, further study in a larger sample size will be more evident to represent the whole Iraqi population.

\section{Study limitation and conclusion}

The present study has a limited sample size which may not represent the complete Iraqi population and would not have included all the probable eye colors. From this study, it was deduced that the IrisPlex system is also suitable for predicting the blue and brown eye color in the small Iraqi population. The prediction of intermediate eye color in the Irisplex system depends on rs1800407A and rs12203592T, and we noticed these two SNPs represent about approximately all samples that wild type 0 and disappeared effective change in both SNPs. In this study, six SNPs were genotyped, which we believe would have affected the phenotypic variance in eye color. Hence, prediction accuracy can be improved if more SNPs are genotyped in the larger Iraqi population. Multi-gene interactions are responsible for the development of eye color. Hence, more exhaustive investigation on newer pigmentation genes in the Iraqi population may further optimize and augment the prediction proficiency of the IrisPlex system. Alike other studies which confirmed the reliability of model-based eye color prediction in diverse ethnic populations, our study also proved the same in a small cohort of Iraqi subjects. However, this does not represent the whole Iraqi population. Hence, further study with a larger Iraqi sample size covering a wider spectrum of genetic SNPs will be more appropriate to compare the sensitivity and specificity of the IrisPlex prediction model with previously published pivotal studies.

\section{Supplementary information}

Supplementary information accompanies this paper at https://doi.org/10. 1186/s41935-020-00200-8.

Additional file 1: Table S1. Primer sequences (PCR) of the six IrisPlex polymorphisms. Table S2. Genotypes for the six currently most eye color-informative SNPs and eye color prediction probabilities for 58 Iraqi volunteers. Table S3. IrisPlex prediction vs observed eye color for all three groups- Blue, Intermediate and Brown. Table S4. Association of gender and eye color prediction accuracy by using the six IrisPlex SNPS.

Additional file 2: Figure S1. Eye color images of 58 Iraqi subjects. Figure S2. Cluster Plots of 6 genetic SNPS.

\section{Abbreviations}

EVCs: Externally visible characters; SNPs: Single nucleotide polymorphisms; AUC: Area under the curve; NPV: Negative predictive value; PPV: Positive predictive value; ROC: Receiver operating characteristics.

\section{Acknowledgements}

The authors are thankful to Yang Hu, Ph.D., CD genomics (USA) for his confirmation about the validity of the results and Safaa K. Kadhem, Ph.D., College of Science, Al Muthanna University for the discussions about the statistical analysis results of this study.

\section{Adherence to national and international regulations} Not applicable.

\section{Authors' contributions}

Nihad A.M. Al-Rashedi contributed to the study conception and design. Nihad A.M. Al-Rashedi and Amar Mousa Mandal contributed to the acquisition of samples. Nihad A.M. Al-Rashedi, Amar Mousa Mandal, and Laith AH Mohamad-Jawad contributed to the analysis and interpretation of the data. Nihad A.M. Al-Rashedi, Amar Mousa Mandal, and Laith AH Mohamad-Jawad contributed to the drafting of the manuscript. Nihad A.M. Al-Rashedi, Amar Mousa Mandal, and Laith AH Mohamad-Jawad contributed to the critical revision of the manuscript. All authors have read and approved the final manuscript.

\section{Funding}

Not applicable.

\section{Availability of data and materials}

All data generated or analyzed during this study are included in this published article and its supplementary information files. 


\section{Ethics approval and consent to participate}

This study was approved by the ethics committee of the Al-Muthanna University (Document number 2032 on 10/5/2018). All participants provided signed informed consent.

\section{Consent for publication}

Given by all the authors.

\section{Competing interests}

The authors declare that they have no competing interests.

\section{Received: 27 November 2019 Accepted: 27 July 2020}

Published online: 11 August 2020

\section{References}

Allwood JS, Harbison S (2013) SNP model development for the prediction of eye colour in New Zealand. Forensic Sci Int Genet 7:444-452. Medline:23597786. https://doi.org/10.1016/j.fsigen.2013.03.005

Branicki W, Brudnik U, Wojas-Pelc A (2009) Interactions between HERC2, OCA2 and MC1R may influence human pigmentation phenotype. Ann Hum Genet 73:160-170. Medline:19208107. https://doi.org/10.1111/j.1469-1809.2009. 00504.x

Branicki W, Liu F, van Duijn K, Draus-Barini J, Pospiech E, Walsh S et al (2011) Model-based prediction of human hair color using DNA variants. Hum Genet 129:443-454. https://doi.org/10.1007/s00439-010-0939-8

Dario P, Mouriño H, Oliveira AR, Lucas I, Ribeiro T, Porto MJ, Costa Santos J, Dias D, Corte Real F (2015) Assessment of IrisPlex-based multiplex for eye and skin color prediction with application to a Portuguese population. Int J Legal Med 129:1191-1200. https://doi.org/10.1007/s00414-015-1248-5

Dembinski GM, Picard CJ (2014) Evaluation of the IrisPlex DNA-based eye color prediction assay in a United States population. Forensic Sci Int Genet 9:111-117. https://doi.org/10.1016/j.fsigen.2013.12.003

Dixon LA, Murray CM, Archer EJ, Dobbins AE, Koumi P, Gill P (2005) Validation of a 21-locus autosomal SNP multiplex for forensic identification purposes, Forensic Sci. Int. 154:62-77

Eiberg H, Troelsen J, Nielsen M, Mukkelsen A, Mengel-From J, Kjaer KW et al (2008) Blue eye color in humans may be caused by a perfectly associated founder mutation in a regulatory element located within the HERC2 gene inhibiting OCA2 expression. Hum Genet 123:177-187. Medline:18172690. https://doi.org/10.1007/s00439-007-0460-x

Franssen L, Coppens JE (2008) Van den Berg Thomas J.T.P. Grading of Iris Color with an Extended Photographic Reference Set. J Opt 1:36-40. https://doi.org/ 10.3921/joptom.2008.36

Freire-Aradas A, Ruiz Y, Phillips C, Maroñas O, Söchtig J, Tato AG, Dios JÁ et al (2014) Exploring iris colour prediction and ancestry inference in admixed populations of South America. Forensic Sci Int Genet 13:3-9. https://doi.org/ 10.1016/j.fsigen.2014.06.007

Frudakis T, Thomas M, Gaskin Z, Venkateswarlu K, Chandra KS, Ginjupalli S et al (2003) Sequences associated with human iris pigmentation. Genetics 165: 2071-2083 Medline:14704187

Han J, Kraft P, Nan H, Guo Q, Chen C, Qureshi A et al (2008) A genome-wide association study identifies novel alleles associated with hair color and skin pigmentation. PLoS Genet 4(5):e1000074. https://doi.org/10.1371/journal. pgen.1000074 PMCID: PMC2367449

Hart KL, Kimura SL, Mushailov V, Budimlija ZM, Prinz M, Wurmbach E (2013) Improved eye- and skin-color prediction based on 8 SNPs. Croat Med J 54: 248-256. Medline:23771755. https://doi.org/10.3325/cmj.2013.54.248

Kastelic V, Pośpiech E, Draus-Barini J, Branicki W, Drobnič K (2013) Prediction of eye color in the Slovenian population using the IrisPlex SNPs. Croat Med J 54(4):381-386

Kayser M, Schneider PM (2009) DNA-based prediction of human externally visible characteristics in forensics: motivations, scientific challenges, and ethical considerations. Forensic Sci Int Genet 3:154-161. https://doi.org/10.1016/j. fsigen.2009.01.012

Liu F, van Duijn K, Vingetling JR, Hofman A, Uitterlinden AG, Janssens ACJW (2009) Eye color and the prediction of complex phenotype from genotypes. Curr Biol 19:R192-3. Medline:19278628. https://doi.org/10.1016/j.cub.2009.01.027

Martinez-Cadenas C, Peña-Chilet M, Ibarrola-Villava M, Ribas G (2013) Gender is a major factor explaining discrepancies in eye colour prediction based on HERC2/OCA2 genotype and the IrisPlex model. Forensic Sci Int Genet 7(4): 453-460. https://doi.org/10.1016/j.fsigen.2013.03.007
Mengel-From J, Brrrsting C, Sanchez JJ, Eiberg H, Morling N (2010) Human eye colour and HERC2, OCA2 and MAT P. Forensic Sci Int Genet 4:323-328. Medline:20457063. https://doi.org/10.1016/j.fsigen.2009.12.004

Pneuman A, Budimlija ZM, Caragine T, Prinz M, Wurmbach E (2012) Verification of eye and skin color predictors in various populations. Leg Med (Tokyo) 14:78-83. Medline:22284939. https://doi.org/10.1016/j.legalmed.2011.12.005

Pośpiech E, Draus-Barini J, Kupiec T, Wojas-Pelc A (2012) Branicki W. Prediction of eye color from genetic data using Bayesian approach. J Forensic Sci 57:880-886 Medline:22372960

Ruiz Y, Phillips C, Gomez-Tato A, Alvarez-Dios J, Casares de Cal M, Cruz R et al (2013) Further development of forensic eye color predictive tests. Forensic Sci Int Genet 7:28-40. Medline:22709892. https://doi.org/10.1016/j.fsigen.2012 05.009

Sanchez J.J., Phillips C., Børsting C., Balogh K., Bogus M., . Fondevila M, Harrison C. D., Musgrave-Brown E., Salas A., Syndercombe-Court D., Schneider P.M., Carracedo A., Morling N. (2006) A multiplex assay with 52 single nucleotide polymorphisms for human identification, Electrophoresis 27:1713-1724.

Spichenok O, Budimlija ZM, Mitchell AA, Jenny A, Kovacevic L, Marjanovic D et al (2011) Prediction of eye color and skin color in diverse populations using seven SNPs. Forensic Sci Int Genet 5:472-478. Medline:21050833. https://doi. org/10.1016/j.fsigen.2010.10.005

Sulem P, Gudbjartsson DF, Stacey SN, Helgason A, Rafnar T, Jakobsdottir M et al (2008) Two newly idnetified genetic determinants of pigmentation in Europeans. Nat Genet 40:835-837. https://doi.org/10.1038/ng.160

Sulem P, Gudbjartsson DF, Stacey SN, Helgason A, Rafnar T, Magnusson KP et al (2007) Genetic determinants of hair, eye and skin pigmentation in Europeans. Nat Genet 39(12):1443-1452. https://doi.org/10.1038/ng.2007.13

Valenzuela RK, Henderson MS, Walsh MH, Garrison NA, Kelch JT, Cohen Barak O, Erickson DT, John Meaney F, Bruce Walsh J, Cheng KC, Ito S, Wakamatsu K, Frudakis T, Thomas M, Brilliant MH (2010) Predicting phenotype from genotype: normal pigmentation. J Forensic Sci 55:315-322

Visser M, Kayser M, Palstra RJ (2012) HERC2 rs12913832 modulates human pigmentation by attenuating chromatin-loop formation between a longrange enhancer and the OCA2 promoter. Genome Res 22:446-455. Medline: 22234890. https://doi.org/10.1101/gr.128652.111

Walsh S, Lindenbergh A, Zuniga SB et al (2011a) Developmental validation of the IrisPlex system: determination of blue and brown iris colour for forensic intelligence. Forensic Sci Int Genet 5:464-471. https://doi.org/10.1016/j.fsigen. 2010.09.008

Walsh S, Liu F, Ballantyne KN et al (2011b) IrisPlex: a sensitive DNA tool for accurate prediction of blue and brown eye colour in the absence of ancestry information. Forensic Sci Int Genet 5:170-180. https://doi.org/10.1016/j.fsigen. 2010.02.004

Walsh S, Wollstein A, Liu F, Chakravarthy U, Rahu M, Seland JH et al (2012) DNAbased eye colour prediction across Europe with the IrisPlex system. Forensic Sci Int Genet 6:330-340. https://doi.org/10.1016/j.fsigen.2011.07.009

Yun L, Gu Y, Rajeevan H, Kidd KK (2014) Application of six IrisPlex SNPS and comparison of two eye color prediction systems in diverse Eurasia populations. Int J Legal Med 128:447-453. https://doi.org/10.1007/s00414013-0953-1

\section{Publisher's Note}

Springer Nature remains neutral with regard to jurisdictional claims in published maps and institutional affiliations. 